\title{
Single-particle density matrix for a time-dependent strongly interacting one-dimensional Bose gas
}

\author{
R. Pezer| \\ Faculty of Metallurgy, University of Zagreb, 44103 Sisak, Croatia \\ T. Gasenzer \\ Institut für Theoretische Physik, Universität Heidelberg, Philosophenweg 16, 69120 Heidelberg, Germany \\ H. Buljan \\ Department of Physics, University of Zagreb, PP 332, Zagreb, Croatia
}

(Dated: June 8, 2018)

\begin{abstract}
We derive a $1 / c$-expansion for the single-particle density matrix of a strongly interacting timedependent one-dimensional Bose gas, described by the Lieb-Liniger model ( $c$ denotes the strength of the interaction). The formalism is derived by expanding Gaudin's Fermi-Bose mapping operator up to $1 / c$-terms. We derive an efficient numerical algorithm for calculating the density matrix for time-dependent states in the strong coupling limit, which evolve from a family of initial conditions in the absence of an external potential. We have applied the formalism to study contraction dynamics of a localized wave packet upon which a parabolic phase is imprinted initially.
\end{abstract}

PACS numbers: 05.30.-d,03.75.Kk

\section{INTRODUCTION}

One of the most attractive many-body quantum systems nowadays has been introduced by Lieb and Liniger in their landmark paper more than forty years ago [1]. The system is composed of $N$ identical $\delta$-interacting bosons in one spatial dimension and is referred to as a Lieb-Liniger (LL) gas. They have presented an explicit form of the many-body wave function for a homogeneous gas with periodic boundary conditions [1], including equations describing the ground state and the excitation spectrum. In the strongly interacting "impenetrablecore" regime [2], such a one dimensional (1D) system is referred to as the Tonks-Girardeau (TG) gas; exact solutions in this limit are obtained by Girardeau's FermiBose mapping [2]. Following Ref. [1], Yang and Yang [3] have eliminated a possible existence of phase transitions in the LL system by proving analyticity of the partition function. After many recent experimental successes [4, 5, 6, 7, 8, 9] in realization of effectively one dimensional (1D) interacting gases, from the weak up to the strongly interacting TG regime [5], the LL model has attracted considerable attention of the physics community. There is a clear reason for this; nontrivial quantum many-body systems are notoriously oblique to a quantitative analysis, and therefore possibility of an exact treatment in particular cases, together with experimental realization, is of great value. Moreover, exact solutions can be useful as a benchmark for approximate treatments aiming to describe a broader range of physical systems.

Even though exact LL many-body wave functions can

*Electronic address: rpezer@phy.hr be constructed in some cases (e.g., stationary 1, 10, 11, $12,13,14,15,16]$ or time-dependent wave functions $[17,18,19,20]$ ), the calculation of observables (correlation functions) from such solutions usually poses a major difficulty in practice $21,22,23,24,25,26,27,28,29$, 30, 31, 32. Various methods have been employed to overcome this difficulty including, for example, the quantum inverse scattering method (e.g., see [21, 29]) and quantum Monte Carlo integration [25]. A recent discussion of several exact methods for the calculation of correlation functions of a nonequilibrium 1D Bose gas can be found in Ref. [32]. In the TG limit, the momentum distribution can be analytically studied for a ring geometry, and also for harmonic confinement (e.g., see [33, 34]). Numerical methods for the calculation of the reduced single-particle density matrix (RSPDM) can be performed efficiently for various TG states (ground state, excited and timedependent states, see Ref. [35] for hard-core bosons on the lattice, and Ref. [36] for the continuous TG model [2]).

Ultracold atoms in 1D atomic wave guides enter the strongly interacting regime at low temperatures, in tight transverse confinement, and with strong effective interactions [37, 38, 39]. The correlations functions of a LL gas can in this limit be calculated by using $1 / c$ expansions (e.g., see [22, 24, 27]) from the TG $(c \rightarrow \infty)$ regime. These calculations in the strongly interacting limit exploit the fact that a bosonic LL gas is dual to a fermionic system [40], such that weakly interacting fermions correspond to strongly interacting bosons and vice versa [40, 41]. A strongly interacting 1D Bose gas was studied in Ref. [42] by using perturbation theory for the dual fermionic system. In Ref. [27], the dynamic structure factor was calculated for zero and finite temperatures.

Here we calculate the $1 / c$ correction for the RSPDM of a Lieb-Liniger gas, which adds upon a recently obtained 
formula for the RSPDM of a TG gas [36]. The method is derived by using the $1 / c$ term of the so-called FermiBose (FB) mapping operator introduced by Gaudin [17]. The FB operator method provides us with exact timedependent solutions of a LL model [17, 19] in the absence of external potentials and other boundary conditions; it was recently used to study free expansion of a LL gas [20]. We derive an efficient numerical algorithm for calculating the RSPDM for time-dependent states in the strong coupling limit, which evolve from a family of initial conditions in the absence of an external potential. We employ it to study the evolution of a many-body wave packet with a parabolic phase imprinted at $t=0$, which corresponds to focusing with a lens in optics, a technique experimentally feasible in 1D atomic gases [9].

This work complements the studies of nonequilibrium dynamics of interacting Bose gases which have been addressed by use of the LL model away from $18,19,20,32$ and in the TG limit [35, 36, 43, 44, 45, 46, 47, 48, 49]. The phenomenological relevance of these studies is underlined by the fact that nonequilibrium dynamics is accessible experimentally [7, 8].

The paper is organized as follows: Section II gives a detailed account of the formalism, where we outline the procedure for the calculation of the RSPDM. In section III we present the example of a Bose gas initially in the ground state within a harmonic trap, effectively in the TG regime; at $t=0$ a parabolic phase is applied on the initial state and the harmonic potential is turned off. The subsequent dynamics leads to focusing of the cloud and local increase of the $1 / c$ correction. Details of the derivation and relevant mathematical identities are collected in the Appendices.

\section{FORMALISM FOR THE TIME-DEPENDENT STRONGLY-INTERACTING LIEB-LINIGER GAS}

The Lieb-Liniger model describes a system of bosons interacting via pointlike interactions; the many-body Schrödinger equation for the LL gas of $N$ such bosons reads [1]

$$
i \frac{\partial \psi_{L L}}{\partial t}=-\sum_{i=1}^{N} \frac{\partial^{2} \psi_{L L}}{\partial x_{i}^{2}}+\sum_{1 \leq i<j \leq N} 2 c \delta\left(x_{i}-x_{j}\right) \psi_{L L}
$$

Here, $\psi_{L L}\left(x_{1}, \ldots, x_{N}, t\right)$ is the time-dependent bosonic wave function, $c$ is the strength of the interaction. For now, we assume the absence of any external potential and boundary conditions. Under these circumstances, the time-dependent LL model (1) can be solved by employing the Fermi-Bose transformation [17, 19]. This method can be applied, e.g., to exactly study free expansion from an initially localized state [20]. If $\psi_{F}\left(x_{1}, \ldots, x_{N}, t\right)$ is an antisymmetric (fermionic) wave function, which obeys the
Schrödinger equation for a noninteracting Fermi gas,

$$
i \frac{\partial \psi_{F}}{\partial t}=-\sum_{i=1}^{N} \frac{\partial^{2} \psi_{F}}{\partial x_{i}^{2}}
$$

then the wave function

$$
\begin{array}{r}
\psi_{L L}\left(x_{1}, \ldots, x_{N}, t\right)=\sqrt{\mathcal{N}(c)} \prod_{1 \leq r<l \leq N}\left[\operatorname{sgn}\left(x_{l}-x_{r}\right)+\right. \\
\left.\frac{1}{c}\left(\frac{\partial}{\partial x_{l}}-\frac{\partial}{\partial x_{r}}\right)\right] \psi_{F}\left(x_{1}, \ldots, x_{N}, t\right)
\end{array}
$$

obeys Eq. (11) as pointed out in Ref. [17] (see also Refs. [19, 20]). Here, $\mathcal{N}(c)$ is a normalization constant, and the differential operator in the square brackets denotes the Fermi-Bose mapping operator [19]. The derivatives do not act on any of the sign functions (the sign functions can be avoided by working in only one sector of the configuration space, e.g., for $x_{1}<x_{2} \ldots<x_{N}$, e.g., see Refs. [19, 20]). We assume that $\psi_{F}\left(x_{1}, \ldots, x_{N}, t\right)$ is normalized to unity. Equation (3) can be reorganized in a finite power series with terms of order $1 / c^{m}$, where $m=0,1, \ldots, N(N-1) / 2$. By using the Fermi-Bose mapping operator in this form, one obtains a systematic expansion of the exact many-body wave function $\psi_{L L}$ in the inverse interaction strength $1 / c$.

The expansion of the wave function (3) in the inverse coupling strength $1 / c$ is particularly useful in the strong coupling limit, as it allows an approximate calculation of one-body observables contained within the reduced single-particle density matrix (RSPDM). In the TonksGirardeau limit, where $c=\infty$, a formula for efficient calculation of the RSPDM has recently been derived [36]. Here we generalize that result to include the $1 / c$ term in the expansion. By keeping only the $1 / c$ terms in the expansion, Eq. (3) reduces to

$$
\begin{gathered}
\psi_{L L}\left(x_{1}, \ldots, x_{N}, t\right) \simeq \sqrt{\mathcal{N}(c)} \prod_{1 \leq i<j \leq N} \operatorname{sgn}\left(x_{j}-x_{i}\right)\left[\psi_{F}+\right. \\
\left.\frac{1}{c} \sum_{1 \leq r<l \leq N} \operatorname{sgn}\left(x_{l}-x_{r}\right)\left(\frac{\partial \psi_{F}}{\partial x_{l}}-\frac{\partial \psi_{F}}{\partial x_{r}}\right)\right]_{\left(x_{1}, \ldots, x_{N}, t\right)}
\end{gathered}
$$

The first term is simply the TG gas wave function [2], while the second term gives the $1 / c$ correction to the TG wave function when the coupling constant $c$ is finite. This expression is the starting point for all results that will be derived in this paper. The RSPDM is defined as

$$
\begin{aligned}
\rho_{L L}(x, y, t)= & N \int d x_{2} \cdots d x_{N} \psi_{L L}\left(x, x_{2}, \ldots, x_{N}, t\right)^{*} \\
& \times \psi_{L L}\left(y, x_{2}, \ldots, x_{N}, t\right) .
\end{aligned}
$$

On inserting Eq. (4) into Eq. (5) ) we obtain a formal 
expression for the $\mathcal{O}(1 / c)$ correction of the RSPDM:

$$
\begin{aligned}
\rho_{L L}(x, y, t)= & \mathcal{N}(c) \hat{I}(X) \psi_{F}^{*}(x, X, t) \psi_{F}(y, X, t) \\
& +\frac{1}{c} \mathcal{N}(c) \sum_{1 \leq r<l \leq N} \hat{I}(X)\left[\operatorname{sgn}\left(x_{l}-x_{r}\right)\right. \\
& \left(\frac{\partial \psi_{F}}{\partial x_{l}}-\frac{\partial \psi_{F}}{\partial x_{r}}\right)_{(x, X, t)}^{*} \psi_{F}(y, X, t) \\
& +\operatorname{sgn}\left(x_{l}-x_{r}\right) \psi_{F}^{*}(x, X, t) \\
& \left.\left(\frac{\partial \psi_{F}}{\partial x_{l}}-\frac{\partial \psi_{F}}{\partial x_{r}}\right)_{(y, X, t)}\right]+\mathcal{O}\left(1 / c^{2}\right) .
\end{aligned}
$$

Here, $X=\left(x_{2}, \ldots, x_{N}\right)$ and the integral operator $\hat{I}(X)$ is defined as:

$$
\hat{I}(X)=N \prod_{n=2}^{N} \int_{-\infty}^{\infty} d x_{n} \operatorname{sgn}\left(x-x_{n}\right) \operatorname{sgn}\left(y-x_{n}\right) .
$$

The first term on the right hand side of Eq. (6) is the TG gas RSPDM [36]. It can be proven (as is done in Appendix (A) that only the partial derivatives with respect to the first coordinate $x_{1}$ in Eq. (6) give a nonvanishing contribution. After eliminating the vanishing terms from Eq. (6) we are left with

$$
\begin{aligned}
\rho_{L L}(x, y, t)=\mathcal{N}(c) \rho_{T G}(x, y, t)+\frac{1}{c} \mathcal{N}(c) & \\
\times & \sum_{l=2}^{N} \hat{I}(X)\left[\operatorname{sgn}\left(x-x_{l}\right)\left(\frac{\partial \psi_{F}}{\partial x_{1}}\right)_{(x, X, t)}^{*} \psi_{F}(y, X, t)+\right. \\
& \left.\operatorname{sgn}\left(y-x_{l}\right) \psi_{F}^{*}(x, X, t)\left(\frac{\partial \psi_{F}}{\partial x_{1}}\right)_{(y, X, t)}\right]+\mathcal{O}\left(1 / c^{2}\right) \\
= & \rho_{T G}(x, y, t)+\frac{1}{c}\left[\eta(x, y, t)+\eta(y, x, t)^{*}\right]+\mathcal{O}\left(1 / c^{2}\right) .
\end{aligned}
$$

In Eq. (8) we have implicitly defined the quantity $\eta(x, y, t)$ :

$$
\begin{array}{r}
\eta(x, y, t)=\sum_{l=2}^{N} \hat{I}(X) \operatorname{sgn}\left(x-x_{l}\right)\left(\frac{\partial \psi_{F}}{\partial x_{1}}\right)_{(x, X, t)}^{*} \psi_{F}(y, X, t) \\
=(N-1) \hat{I}(X) \operatorname{sgn}\left(x-x_{2}\right)\left(\frac{\partial \psi_{F}}{\partial x_{1}}\right)_{(x, X, t)}^{*} \psi_{F}(y, X, t) .
\end{array}
$$

By using the calculation presented in Appendix $\mathrm{A}$ it follows that

$$
\int d x\left[\eta(x, x, t)+\eta(x, x, t)^{*}\right]=0,
$$

that is, the $1 / c$ correction to the single-particle density $\rho_{L L}(x, x, t)$ increases the density in some regions of space, but also lowers it in others such that the integral over the terms of order $1 / c$ is zero. By using this result we see that in the leading $1 / c$ approximation we can take $\mathcal{N}(c)=1$; this fact has already been utilized in the last line of Eq. (8). It is straightforward to verify that the integrals $\hat{I}(X) \operatorname{sgn}\left(x-x_{l}\right)\left(\frac{\partial \psi_{F}}{\partial x_{1}}\right)_{(x, X)}^{*} \psi_{F}(y, X)$ are independent of $l(2 \leq l \leq N)$, which yields the second identity in Eq. (9). The last line of Eq. (8) verifies that to order $1 / c$ the RSPDM possesses as required the symmetry $\rho_{L L}(x, y, t)=\rho_{L L}(y, x, t)^{*}$.

Up to this point we did not make any assumptions on the structure of $\psi_{F}$ (except that it is antisymmetric and normalized), that is, the derivation was general, valid even if the wave functions should describe the gas in an external potential etc. Quite generally, $\psi_{F}$ can also be considered as a function of $c$, and one could expand it in powers of $1 / c$. ¿From this point on, $\psi_{F}$ will be represented as a Slater determinant formed from single particle wave functions,

$$
\psi_{F}=\frac{1}{\sqrt{N !}} \sum_{P}(-)^{P} \phi_{P 1}\left(x_{1}, t\right) \cdots \phi_{P N}\left(x_{N}, t\right)
$$

such wave functions can be used to study dynamics on an infinite line, which arises from initial conditions given by Eqs. (44) and (10). In Eq. (10), $\phi_{j}(x, t)(j=1, \ldots, N)$ are orthonormal single-particle wave functions which obey the Schrödinger equation $i \partial \phi_{j} / \partial t=-\partial^{2} \phi_{j} / \partial x^{2} . P$ denotes a permutation $P(1, \ldots, N)=(P 1, \ldots, P N)$ of the particle number indices, and $(-)^{P}$ is its signature. In this form $\psi_{F}$ enables us to rewrite Eq. (8) such that it involves 1D integrals only, resulting in certain algebraic cofactors suitable for numerical calculation.

\section{A. Algorithm for RSPDM calculation}

For the sake of clarity of the presentation, we first describe the algorithm for calculation of the RSPDM, and only afterwards provide its derivation. Without loss of generality we consider the case $x<y$. The first step is to calculate the integrals

$$
I_{k, l}(x, y, t)=\delta_{k l}-2 \int_{x}^{y} d x^{\prime} \phi_{k}^{*}\left(x^{\prime}, t\right) \phi_{l}\left(x^{\prime}, t\right)
$$

and

$$
\bar{I}_{k, l}(y, t)=-\delta_{k l}+2 \int_{-\infty}^{y} d x^{\prime} \phi_{k}^{*}\left(x^{\prime}, t\right) \phi_{l}\left(x^{\prime}, t\right)
$$

for $k, l=1, \ldots, N$. 
These integrals are arranged in the following matrices:

$$
\mathbf{P}(x, y, t)=\left[\begin{array}{cccc}
I_{1,1}(x, y, t) & I_{1,2}(x, y, t) & \ldots & I_{1, N}(x, y, t) \\
I_{2,1}(x, y, t) & I_{2,2}(x, y, t) & \ldots & I_{2, N}(x, y, t) \\
\vdots & \vdots & \ddots & \vdots \\
I_{N, 1}(x, y, t) & I_{N, 2}(x, y, t) & \ldots & I_{N, N}(x, y, t)
\end{array}\right]
$$

and

$$
\mathbf{P}^{(l)}(x, y, t)=\left[\begin{array}{cccccc}
I_{1,1}(x, y, t) & I_{1,2}(x, y, t) & \ldots & \bar{I}_{1, l}(y, t) & \ldots & I_{1, N}(x, y, t) \\
I_{2,1}(x, y, t) & I_{2,2}(x, y, t) & \ldots & \bar{I}_{2, l}(y, t) & \ldots & I_{2, N}(x, y, t) \\
\vdots & \vdots & & \ddots & & \vdots \\
I_{N, 1}(x, y, t) & I_{N, 2}(x, y, t) & \ldots & \bar{I}_{N, l}(y, t) & \ldots & I_{N, N}(x, y, t)
\end{array}\right]
$$

Let us define the column vector

$$
\boldsymbol{\Psi}(x, t)=\left(\begin{array}{c}
\phi_{1}(x, t) \\
\vdots \\
\phi_{N}(x, t)
\end{array}\right)
$$

and its first spatial derivative

$$
\boldsymbol{\Psi}^{\prime}(x, t)=\left(\begin{array}{c}
\phi_{1}^{\prime}(x, t) \\
\vdots \\
\phi_{N}^{\prime}(x, t)
\end{array}\right)
$$

The TG reduced single-particle density matrix is given by 36 ]

$$
\begin{aligned}
\rho_{T G}(x, y, t)=\operatorname{det}[\mathbf{P}(x, y, t)] \\
\boldsymbol{\Psi}^{\dagger}(x, t)\left[\mathbf{P}(x, y, t)^{-1}\right]^{T} \mathbf{\Psi}(y, t) .
\end{aligned}
$$

The quantity $\eta(x, y, t)$ can also be written in a convenient matrix form (suitable for efficient numerical implementation):

$$
\begin{aligned}
\eta(x, y, t)=\sum_{l=1}^{N}\{\operatorname{det} & {\left.\left[\mathbf{P}^{(l)}(x, y, t)\right] \mathbf{\Psi}^{\prime \dagger}(x, t)\left[\mathbf{P}^{(l)}(x, y, t)^{-1}\right]^{T} \mathbf{\Psi}(y, t)\right\} } \\
& -\operatorname{det}[\mathbf{P}(x, y, t)] \mathbf{\Psi}^{\prime \dagger}(x, t)\left[\mathbf{P}(x, y, t)^{-1}\right]^{T} \mathbf{\Psi}(y, t)
\end{aligned}
$$

If any of the matrices $\mathbf{P}^{(l)}$ happen to be singular, we can resort to a direct calculation via algebraic cofactors (see the proof of the algorithm in Appendix B). This happens rarely and only for some particular high-symmetry points. Equations (13)-(18) provide the grounds for an efficient numerical method for calculating the RSPDM, which is a generalization of the previously introduced method for the TG gas 36].

It is convenient to calculate the diagonal correction to the RSPDM. In this case, the matrices appearing in Eq. (18) are very simple and it is straightforward to obtain

$$
\begin{aligned}
c\left[\frac{\rho_{L L}(x, x, t)}{\mathcal{N}(c)}-\right. & \left.\rho_{T G}(x, x, t)\right] \simeq \\
& {\left[\operatorname{Tr}\left(\rho^{\prime}\right) \operatorname{Tr}(\bar{I})-\operatorname{Tr}\left(\rho^{\prime} \cdot \bar{I}\right)\right]_{(x, t)} }
\end{aligned}
$$

where on the right hand side $\rho$ is $N \times N$ matrix given by

$$
\rho_{k, l}(x, t)=\phi_{k}^{*}(x, t) \phi_{l}(x, t)
$$

and primes denote the first spatial derivative. We can also omit the normalisation constant from Eq. (19) since $\mathcal{N}(c)=1$ within the $1 / c$ approximation considered here.

\section{CONTRACTION DYNAMICS OF STRONGLY INTERACTING 1D BOSE GAS}

In this section we apply the presented formalism to investigate time-evolution of a many-body wave packet which contracts in space after a parabolic phase was imprinted on it. More specifically, we consider $N$ strongly interacting bosons which are initially in the ground state in the presence of a harmonic potential. We assume that this initial state is well described by the Tonks-Girardeau ground state wave function, a symmetrized Slater determinant. As described in more detail below, at time $t=0$, a parabolic phase is suddenly imprinted onto this state, and the harmonic potential is turned off. Imprinting the 
parabolic phase is experimentally feasible [9] and is equivalent to the action of a focusing lens on the travellingwave state of a light beam in optics. In the context of atomic gases this can be achieved by applying, over a short period of time, a tight longitudinal harmonic potential to the one-dimensional gas. This results in providing different initial momenta to different parts of the wave packet, with the momenta being proportional to the distance from the center of the wave packet and directed towards the center.

In order to connect the model written in Eq. (11) to physical units we first write the 1D Schrödinger equation for this system:

$$
\begin{aligned}
i \hbar \frac{\partial \psi_{L L}}{\partial \tau}=\sum_{i=1}^{N}\left[-\frac{\hbar^{2}}{2 m} \frac{\partial^{2}}{\partial \xi_{i}^{2}}\right. & \left.+\frac{m \omega_{H O}^{2}}{2} \xi_{i}^{2}\right] \psi_{L L} \\
& +\sum_{1 \leq i<j \leq N} 2 g \delta\left(\xi_{i}-\xi_{j}\right) \psi_{L L}
\end{aligned}
$$

where $g$ is the $1 \mathrm{D}$ scattering length [37], $\xi$ is the spatial coordinate, and $\tau$ denotes time. Here we denote the axial trapping frequency by $\omega_{H O}$. By multiplying the above expression with $2 / \hbar \omega_{H O}$ we obtain a dimensionless equation suitable for numerical calculation and a physical interpretation of the relevant scales:

$$
\begin{aligned}
i \frac{\partial \psi_{L L}}{\partial t}= & \sum_{i=1}^{N}\left[-\frac{\partial^{2}}{\partial x_{i}^{2}}+x_{i}^{2}\right] \psi_{L L} \\
& +\sum_{1 \leq i<j \leq N} 2 \frac{2 g}{a_{H O} E_{H O}} \delta\left(x_{i}-x_{j}\right) \psi_{L L}
\end{aligned}
$$

where we have denoted zero point energy of the harmonic trap with $E_{H O}=\frac{\hbar \omega_{H O}}{2}$. The unit of time is $2 / \omega_{H O}$, while space is in units $a_{H O}=\sqrt{\hbar / m \omega_{H O}}$; in other words $x_{i}=\xi_{i} / a_{H O}$, and $t=\omega_{H O} \tau / 2$. If we set $c=2 g / a_{H O} E_{H O}$ we easily verify that it is exactly the interaction strength of the LL model $c$, see Eq. (1). For concreteness, let us assume that the harmonic trap has frequency $\omega_{H O}=60 \mathrm{~Hz}$, which is loaded with ${ }^{87} \mathrm{Rb}$ atoms, $N=12$. These values yield $a_{H O}=1.38 \mu \mathrm{m}$. As we have already stated, we assume that the effective interaction strength $c$ is sufficiently large such that the system is initially in the Tonks-Girardeau regime, and that the $1 / c$ correction is negligible at $t=0$. Under these conditions the ground state is given by

$$
\psi_{g . s .}=\left[\prod_{1 \leq i<j \leq N} \operatorname{sgn}\left(x_{j}-x_{i}\right)\right] \underset{j, m=1}{N}\left[\phi_{j}\left(x_{m}\right)\right] / \sqrt{N !}
$$

where $\phi_{j}(x)$ is the $j$ th eigenstate of the SP harmonic oscillator $-\phi_{j}^{\prime \prime}+x^{2} \phi_{j}=E_{j} \phi_{j}$. At $t=0$ we imprint a parabolic phase to the system. Technically, each of the $\mathrm{SP}$ wave functions in the determinant (21) is multiplied by

$$
P(x)=e^{-i(x / b)^{2}}
$$

where we have chosen $b=\sqrt{2}$. Thus, the initial wave function which starts to evolve in the absence of a trapping potential is

$$
\begin{aligned}
\psi_{L L}\left(x_{1}, \ldots, x_{N}, 0\right) \approx & {\left[\prod_{1 \leq i<j \leq N} \operatorname{sgn}\left(x_{j}-x_{i}\right)\right] } \\
& \operatorname{det}_{j, m=1}^{N}\left[\phi_{j}\left(x_{m}\right) P\left(x_{m}\right)\right] / \sqrt{N !} .
\end{aligned}
$$

After this initial excitation the wave packet starts to contract. Figure 1 displays the evolution of the SP $x$-space density and the momentum distribution (SP $k$-space density) for $c=50$; the momentum distribution is defined as 33.

$$
n_{L L}(k, t)=(2 \pi)^{-1} \int_{-\infty}^{\infty} d x \int_{-\infty}^{\infty} d x^{\prime} e^{-i k\left(x-x^{\prime}\right)} \rho_{L L}\left(x, x^{\prime}, t\right) .
$$

It should be emphasized that the results are scalable, that is, the same results can be obtained for smaller values of $c$ provided that the initial state is broader (this corresponds to the fact that for the LL gas in equilibrium the regime is determined by the interaction strength $c$ divided by the linear density [1]). As the gas becomes more dense during the contraction, the $1 / c$ correction to the TG density becomes larger and clearly visible in the $x$-space density. Note that the gas compresses more strongly for the finite $c$ in comparison to the TG results. After the wave packet reaches the maximally dense configuration, it starts to freely expand and the $1 / c$ correction starts to become less visible. It is interesting to note that while the changes in the $x$-space density are nonnegligible in contraction, the $1 / c$ correction in the $k$ space density is practically negligible at all times. ¿From our simulations we find quite generally that the $1 / c$ correction to the $k$-space density is much less sensitive to changes in the coupling strength than the $x$-space density. Note that the total $x$-space density in the presented form of the $1 / c$ approximation can become negative in some parts of the space, if the approximation is used beyond its range of validity (e.g., if c is not sufficiently large). This gives a clear indication that higher order terms, of order $\mathcal{O}\left(1 / c^{2}\right)$, become relevant, at least in those parts of the space where the density is negative.

We emphasize again that the results presented here, for an evolution in one dimension without further boundary conditions, are correct up to $1 / c$ for all times of the evolution, i.e., there is no accumulation of the error in time due to the approximation and we can extract the asymptotic behaviour of the LL gas.

\section{CONCLUSION}

We have studied the $1 / c$ expansion in the strong coupling limit of the Lieb-Liniger model, exploiting the formalism based on a mapping between free Fermi and interacting Bose gas. More specifically we have developed an 

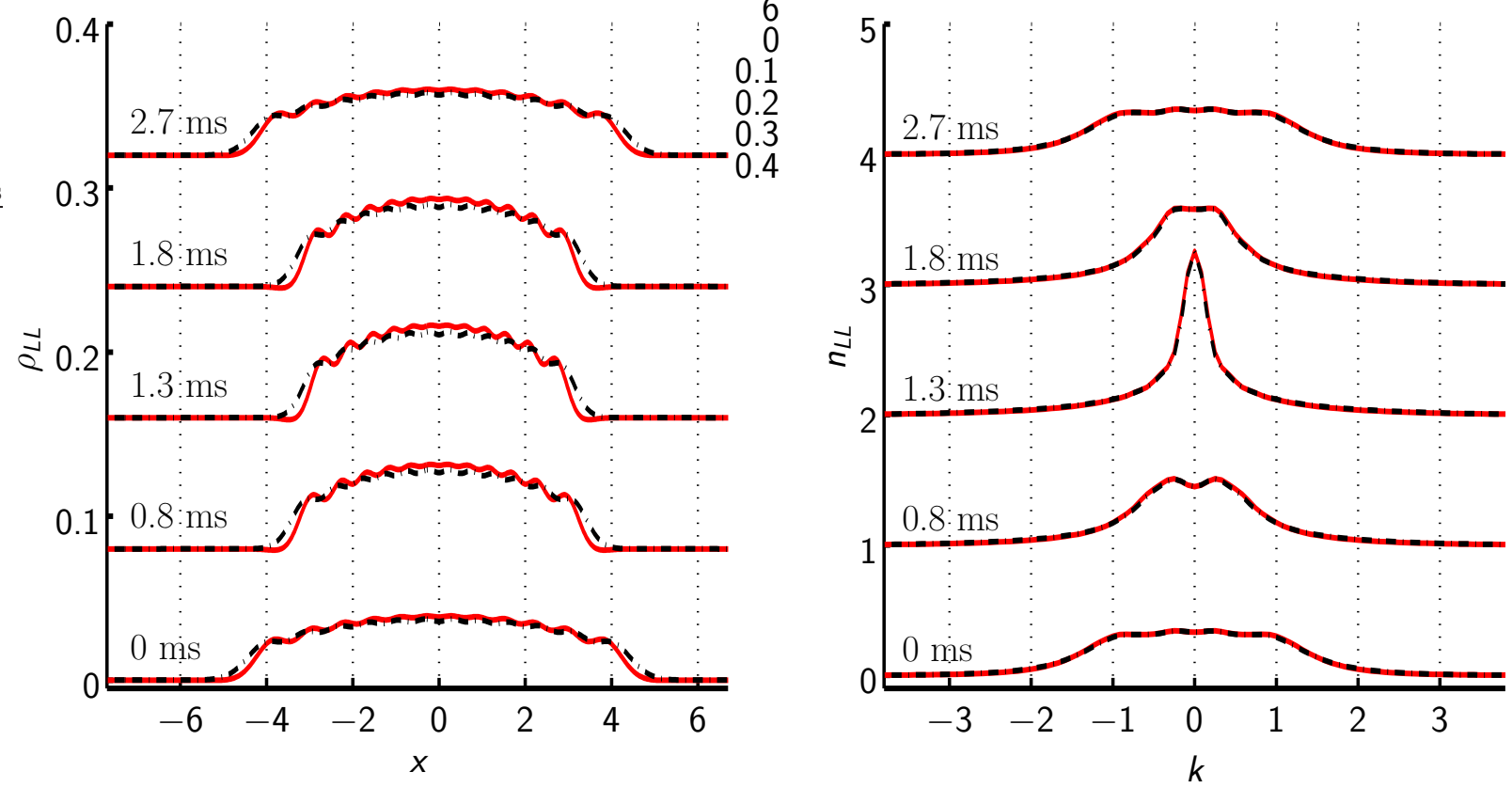

FIG. 1: (color online) The $x$-space density (left, red solid line), and momentum distribution (right, red solid line) of the LL gas with $\mathrm{N}=12$ bosons and $c=50$ at different times of the propagation. At the time of 1.3 ms the cloud attains the maximal compression. The corresponding shapes for the TG gas are also shown as black dot-dashed lines. The difference between red solid and black dot-dashed lines corresponds to the $1 / c$ correction. The curves are shifted on the ordinate axis for better visibility. See text for details.

efficient algorithm to calculate the reduced one body density matrix of the strongly interacting LL gas, which is based on the method previously developed for the TonksGirardeau gas [36], and the Fermi-Bose transformation [17, 19, 20]. The method is suitable to describe dynamics on an infinite line, in the absence of external potentials, and for initial states which can be described by Equations (41) and (10). An attractive feature is stable accuracy for all times since the error is not being accumulated during the evolution. For the $x$-space density we provide a very compact formula Eq. (19) that can be employed for quite large numbers of particles enabling fast calculation of the nonequilibrium $x$-space density dynamics. In addition, the efficiency of the method is demonstrated in an example discussed in Sec. [II] we have examined nonequilibrium dynamics where a parabolic phase is imprinted onto a localized wave packet after which the wave packet contracts for a while. Our simulation studies the setup which was experimentally realized in Ref. 9] for a weakly interacting Bose gas at finite temperature.

\section{Acknowledgments}

R.P. and H.B. acknowledge support from the Croatian Ministry of Science (Grant No. 119-0000000-1015). T.G. acknowledges support by the Deutsche Forschungsgemeinschaft. This work is also supported in part by the Croatian-German scientific collaboration funded by DAAD and MZOŠ, and the Croatian National Founda- tion for Science.

\section{APPENDIX A: FORMULAS}

Integrals in Eq. (6) involving derivatives in integration coordinates all vanish due to following expression for $k \neq$ 1 and for any $l \neq k$ :

$$
\begin{aligned}
& \hat{I}(X) \operatorname{sgn}\left(x_{k}-x_{l}\right)\left(\frac{\partial \psi_{F}}{\partial x_{k}}\right)_{(x, X)}^{*} \psi_{F}(y, X) \\
& +\hat{I}(X) \operatorname{sgn}\left(x_{k}-x_{l}\right) \psi_{F}^{*}(x, X)\left(\frac{\partial \psi_{F}}{\partial x_{k}}\right)_{(y, X)} \\
& =\hat{I}(X) \operatorname{sgn}\left(x_{k}-x_{l}\right) \frac{\partial}{\partial x_{k}}\left[\psi_{F}^{*}(x, X) \psi_{F}(y, X)\right]
\end{aligned}
$$

This is obtained by collecting the corresponding terms from the first and the second part of the $1 / c$ correction to the RSPDM in Eq. (6). Let us now look at the $k$ th integration only. Integrating by parts we have:

$$
\begin{array}{r}
{\left.\left[\operatorname{sgn}\left(x_{k}-x_{l}\right) \psi_{F}^{*}(x, X) \psi_{F}(y, X)\right]\right|_{-\infty} ^{\infty}-} \\
\int_{-\infty}^{\infty} d x_{k} 2 \delta\left(x_{k}-x_{l}\right) \psi_{F}^{*}(x, X) \psi_{F}(y, X)
\end{array}
$$

The first term is zero due to boundary condition at infinity and the second term is zero because of the antisymmetry of $\psi_{F}$. By using the method presented above it is 
straightforward to see that

$$
\int_{-\infty}^{\infty} d x\left[\eta(x, x, t)+\eta(x, x, t)^{*}\right]=0
$$

where $\eta$ is defined in Eq. (9). A direct consequence is that the normalization constant $\mathcal{N}(c)=1$ in the $1 / c$ approximation cosidered here.

\section{APPENDIX B: DETERMINANT EXPANSION}

In this appendix we derive Eq. (18). The derivation is similar to that for the RSPDM of a TG gas [36]. To simplify the notation, we will suppress the time variable in all formulas. By inserting Eq. (10) for $\psi_{F}$ in Eq. (9) for $\eta$ we obtain

$$
\begin{aligned}
\eta(x, y)= & \frac{N(N-1)}{N !} \sum_{i j}(-)^{i+j}\left[\phi_{i}^{\prime}(x)\right]^{*} \phi_{j}(y) \\
& \sum_{P, Q}(-)^{P}(-)^{Q} \bar{I}_{p_{2}, q_{2}} I_{p_{3}, q_{3}} \ldots I_{p_{N}, q_{N}} .
\end{aligned}
$$

Here, $P \in S_{N-1}$ is a permutation of the $N-1$ indices not including $i$ : $\left(p_{2}, \ldots, p_{N}\right)=P(1 \ldots i-1 i+1 \ldots N)$. Similarly, $Q \in S_{N-1}$ permutes the $N-1$ indices not including $j:\left(q_{2}, \ldots, q_{N}\right)=Q(1 \ldots j-1 j+1 \ldots N)$.

We consider the sum over the permutations. Let $\left(q_{3}^{\prime \prime}, \ldots, q_{N}^{\prime \prime}\right)$ be the ordered series of integers $\{1, \ldots, N\} \backslash\{l, j\}$, where $l \neq j$, and $Q^{\prime} \in S_{N-2}$ a permutation of these $N-2$ numbers, $\left(q_{3}^{\prime}, \ldots, q_{N}^{\prime}\right)=$
$Q^{\prime}\left(q_{3}^{\prime \prime}, \ldots, q_{N}^{\prime \prime}\right)$. With these, the sum over permutations in Eq. (B1) can be written as

$$
\begin{aligned}
& \frac{1}{(N-2) !} \sum_{P, Q}(-)^{P+Q} \bar{I}_{p_{2}, q_{2}} I_{p_{3}, q_{3}} \cdots I_{p_{N}, q_{N}} \\
= & \frac{1}{(N-2) !} \sum_{\substack{l=1 \\
(l \neq j)}}^{N}(-)^{l+1} \sum_{P, Q^{\prime}}(-)^{P+Q^{\prime}} \bar{I}_{p_{2}, l} I_{p_{3}, q_{3}^{\prime}} \cdots I_{p_{N}, q_{N}^{\prime}} \\
= & \sum_{\substack{l=1 \\
l \neq j}}^{N} \sum_{P}(-)^{l+1}(-)^{P} \bar{I}_{p_{2}, l} I_{p_{3}, q_{3}^{\prime \prime}} \cdots I_{p_{N}, q_{N}^{\prime \prime}} \\
= & \sum_{\substack{l=1 \\
(l \neq j)}}^{N} \operatorname{det} \mathbf{P}_{i, j}^{(l)}(x, y) .
\end{aligned}
$$

Here, $\mathbf{P}_{i, j}^{(l)}$ is a $N-1 \times N-1$ matrix obtained by eliminating the $i$ th row and the $j$ th column from the matrix $\mathbf{P}^{(l)}$ defined in Eq. (13). This yields the following result for $\eta(x, y)$ :

$$
\eta(x, y)=\sum_{i j}\left[\phi_{i}^{\prime}(x)\right]^{*} \phi_{j}(y) \sum_{\substack{l=1 \\ l \neq j}}(-)^{i+j} \operatorname{det} \mathbf{P}_{i, j}^{(l)}(x, y)
$$

The final result, Eq. (18), is obtained from (B3) with the use of standard matrix algebra connecting minors, the cofactor matrix, and the matrix inverse.
[1] E. Lieb and W. Liniger, Phys. Rev. 130, 1605 (1963); E. Lieb, Phys. Rev. 130, 1616 (1963).

[2] M. Girardeau, J. Math. Phys. 1, 516 (1960).

[3] C.N. Yang and C.P. Yang, J. Math. Phys. 10, 1115 (1969);

[4] F. Schreck, L. Khaykovich, K.L. Corwin, G. Ferrari, T. Bourdel, J. Cubizolles, and C. Salomon, Phys. Rev. Lett. 87, 080403 (2001); A. Görlitz, J.M. Vogels, A.E. Leanhardt, C. Raman, T.L. Gustavson, J.R. Abo-Shaeer, A.P. Chikkatur, S. Gupta, S. Inouye, T. Rosenband, and W. Ketterle, ibid. 87, 130402 (2001); M. Greiner, I. Bloch, O. Mandel, T.W. Hänsch, and T. Esslinger, ibid. 87, 160405 (2001); H. Moritz, T. Stöferle, M. Kohl, and T. Esslinger, ibid. 91, 250402 (2003); B. Laburthe-Tolra, K.M. O'Hara, J.H. Huckans, W.D. Phillips, S.L. Rolston, and J.V. Porto, ibid. 92, 190401 (2004); T. Stöferle, H. Moritz, C. Schori, M. Kohl, and T. Esslinger, ibid. 92, 130403 (2004).

[5] T. Kinoshita, T. Wenger, and D.S. Weiss, Science 305, 1125 (2004); B. Paredes, A. Widera, V. Murg, O. Mandel, S. Fölling, I. Cirac, G. V. Shlyapnikov, T. W. Hänsch, and I. Bloch, Nature (London) 429, 277 (2004).

[6] C.D. Fertig, K.M. O'Hara, J.H. Huckans, S.L. Rolston, W.D. Phillips, and J.V. Porto, Phys. Rev. Lett. 94, 120403 (2005).
[7] T. Kinoshita, T. Wenger, and D.S. Weiss, Nature (London) 440, 900 (2006).

[8] S. Hofferberth, I. Lesanovsky, B. Fischer, T. Schumm and J. Schmiedmayer, Nature 449, 324 (2007).

[9] A. H. van Amerongen, J. J. P. van Es, P. Wicke, K. V. Kheruntsyan, and N. J. van Druten, Phys. Rev. Lett. 100, 090402 (2008).

[10] J.B. McGuire, J. Math. Phys. 5, 622 (1964).

[11] M. Gaudin, Phys. Rev. A 4, 386 (1971).

[12] J.G. Muga and R.F. Snider, Phys. Rev. A 57, 3317 (1998).

[13] K. Sakmann, A.I. Streltsov, O.E. Alon, and L.S. Cederbaum, Phys. Rev. A 72, 033613 (2005).

[14] M.T. Batchelor, X.-W. Guan, N. Oelkers, and C. Lee, J. Phys. A 38, 7787 (2005).

[15] Y. Hao, Y. Zhang, J.Q. Liang, and S. Chen, Phys. Rev. A 73, 063617 (2006).

[16] A.G. Sykes, P.D. Drummond, and M.J. Davis, Phys. Rev. A 76, 063620 (2007).

[17] M. Gaudin, La function d'Onde de Bethe (Paris, Masson, 1983).

[18] M.D. Girardeau, Phys. Rev. Lett. 91, 040401 (2003).

[19] H. Buljan, R. Pezer, and T. Gasenzer, Phys. Rev. Lett. 100, 080406 (2008); 102, 049903(E) (2009).

[20] D. Jukić, R. Pezer, T. Gasenzer, and H. Buljan, Phys. 
Rev. A 78, 053602 (2008); D. Jukić, B. Klajn, and H. Buljan, Phys. Rev. A 79, 033612 (2009).

[21] V.E. Korepin, N.M. Bogoliubov, and A.G. Izergin, Quantum Inverse Scattering Method and Correlation Functions (Cambridge, Cambridge University Press, 1993).

[22] M. Jimbo and T. Miwa, Phys. Rev. D 24, 3169 (1981).

[23] M. Olshanii and V. Dunjko, Phys. Rev. Lett. 91, 090401 (2003).

[24] D.M. Gangardt and G.V. Shlyapnikov, New J. of Phys. 5, 79 (2003).

[25] G.E. Astrakharchik and S. Giorgini, Phys. Rev. A 68, 031602(R) (2003).

[26] K.V. Kheruntsyan, D.M. Gangardt, P.D. Drummond, and G.V. Shlyapnikov, Phys. Rev. A 71, 053615 (2005).

[27] J. Brand and A.Yu. Cherny, Phys. Rev. A 72, 033619 (2005); A.Yu. Cherny and J. Brand, Phys. Rev. A 79, 023612 (2006).

[28] P.J. Forrester, N.E. Frankel, and M.I. Makin, Phys. Rev. A 74, 043614 (2006).

[29] J.-S. Caux, P. Calabrese, and N. A. Slavnov, J. Stat. Mech. (2007) P01008.

[30] P. Calabrese and J.-S. Caux, Phys. Rev. Lett. 98, 150403 (2007).

[31] A. Imambekov and L.I. Glazman, Phys. Rev. Lett. 100, 206805 (2008)

[32] V. Gritsev, T. Rostunov, and E. Demler, arXiv:0904.3221 1 .

[33] A. Lenard, J. Math. Phys. 5, 930 (1964).

[34] P.J. Forrester, N.E. Frankel, T.M. Garoni, and N.S. Witte, Phys. Rev. A 67, 043607 (2003).
[35] M. Rigol and A. Muramatsu, Phys. Rev. Lett. 94, 240403 (2005); ibid. Mod. Phys. Lett. B 19, 861 (2005).

[36] R. Pezer and H. Buljan, Phys. Rev. Lett. 98, 240403 (2007).

[37] M. Olshanii, Phys. Rev. Lett. 81, 938 (1998).

[38] D.S. Petrov, G.V. Shlyapnikov, and J.T.M. Walraven, Phys. Rev. Lett. 853745 (2000).

[39] V. Dunjko, V. Lorent, and M. Olshanii, Phys. Rev. Lett. 865413 (2001).

[40] T. Cheon and T. Shigehara, Phys. Rev. Lett. 82, 2536 (1999).

[41] V.I. Yukalov and M.D. Girardeau, Laser Phys. Lett 2, 375 (2005).

[42] D. Sen, J. Phys. A: Math. Gen. 36 7517-7531 (2003).

[43] M.D. Girardeau and E.M. Wright, Phys. Rev. Lett. 84, 5691 (2000).

[44] M.D. Girardeau and E.M. Wright, Phys. Rev. Lett. 84 5239 (2000).

[45] A. Minguzzi and D.M. Gangardt, Phys. Rev. Lett. 94, 240404 (2005).

[46] A. del Campo and J.G. Muga, Europhys. Lett. 74, 965 (2006).

[47] M. Rigol, V. Dunjko, V, Yurovskii, and M. Olshanii, Phys. Rev. Lett. 98, 050405 (2007).

[48] H. Buljan, O. Manela, R. Pezer, A. Vardi, and M. Segev, Phys. Rev. A 74, 043610 (2006).

[49] D.M. Gangardt and M. Pustilnik, Phys. Rev. A 77, 041604(R) (2008). 